\title{
APRECIACIÓN EN LOS RESULTADOS DE LAS PRUEBAS SABER PRO'
}

\section{APPRECIATION OF THESABER PRO TEST RESULTS}

\author{
Derly Francedy Poveda Pineda ${ }^{2}$ \\ José Eriberto Cifuentes Medina ${ }^{3}$ \\ José Antonio Chacón Benavides ${ }^{4}$
}

Universidad Pedagógica y Tecnológica de

Colombia

\section{RESUMEN}

Se presenta el análisis de los resultados de

$1 \quad$ Artículo de investigación. Se deriva del proyecto "Análisis de los resultados de las pruebas saber pro en la licenciatura en educación básica" con código SGI 2966 y del proyecto: "Impacto del plan de acompañamiento académico Hércules" con código SGI 2989.

$2 \quad$ Magíster en Elearning, Universidad Autónoma de Bucaramanga, Especialista en Gerencia Educacional y Licenciada en Informática Educativa, Universidad Pedagógica y Tecnológica de Colombia. Facultad de Estudios a Distancia. Integrante grupo de Investigación SIEK. Profesorainvestigadora Licenciatura en Educación Básica Primaria, Universidad Pedagógica y Tecnológica de Colombia. . Orcid: https://orcid.org/0000-0002-1616-112X Contacto: derly. poveda@uptc.edu.co

3 Investigador Asociado (I) SNCTel, 833/2018. Magister en Educación, Especialista en Evaluación Educativa, Licenciado en Teología, Licenciado en Filosofía y Educación Religiosa, Universidad Santo Tomás. Especialista en Pedagogía y Docencia, Licenciado en Ciencias Sociales, Fundación Universitaria del Área Andina. Integrante grupo de Investigación SIEK. Profesor-investigador de la Licenciatura en Educación Básica Primaria, Facultad de Estudios a Distancia, Universidad Pedagógica y Tecnológica de Colombia. Orcid: https://orcid.org/0000-0001-5702-620X Contacto: joseeriberto.cifuentes@uptc.edu.co

$4 \quad$ Magister en Administración y Planificación Educativa, Especialista en Educación Personalizada, Licenciado en Ciencias de la Educación Física y Matemáticas. Integrante grupo de Investigación SIEK. Profesor-investigador de la Licenciatura en Educación Básica Primaria, Facultad de Estudios a Distancia, Universidad Pedagógica y Tecnológica de Colombia. Orcid: https://orcid.org/0000-0002-8515-7386 Contacto: jose.chacon@uptc.edu.co

los estudiantes pertenecientes al programa de Licenciatura en Educación Básica con énfasis en Matemáticas, HumanidadesyLengua Castellana, de la Universidad Pedagógica y Tecnológica de Colombia - UPTC. La investigación describe la participación de 320 estudiantes de los Centros Regionales de Educación a Distancia - CREAD, de Tunja, Duitama, Sogamoso, Chiquinquirá y Bogotá; donde un $75 \%$ es de género femenino y un $25 \%$ de género masculino. El estudio conlleva a realizar un análisis de los resultados de las pruebas estandarizadas que presentaron los futuros licenciados en Competencias Genéricas: Comunicación Escrita, Razonamiento Cuantitativo, Lectura Crítica, Ingles y Competencias Ciudadanas, las cuales presentan también otros estudiantes de distintos programas, que están próximos a graduarse de su formación profesional. En tanto, el análisis llevado a cabo, está correlacionado a las competencias específicas de la formación de licenciados; competencias que se enmarcan en: Enseñar, Evaluar y Formar; componentes propios de un profesional en educación y con especificidad en educación básica. Se realiza la comparación entre el programa, la 
institución, la sede y el grupo de referencia, que permitió consolidar un estudio descriptivo y una aproximación a los resultados obtenidos por los estudiantes del programa.

\section{PALABRAS CLAVE}

$\begin{array}{ccc}\text { Pruebas } & \text { Estandarizadas, } & \text { Competencias } \\ \text { Genéricas, } & \text { Competencias } & \text { Específicas, }\end{array}$ Educación Básica, Licenciado

\section{ABSTRACT}

The analysis of the results of the Saber Pro tests, for the year 2020, of the students belonging to the Bachelor of Basic Education program with an emphasis on Mathematics, Humanities and Spanish Language, of the Pedagogical and Technological University of Colombia - UPTC, is presented. The research describes the participation of 320 students from the Regional Centers for Distance Education - CREAD, from Tunja, Duitama, Sogamoso, Chiquinquirá and

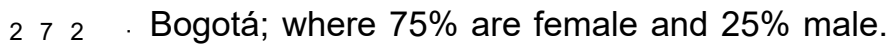
The study leads to an analysis of the results of the standardized tests presented by future graduates in Generic Competences: Written Communication, Quantitative Reasoning, Critical Reading, English and Citizen Competences, which are also presented by other students from different programs, who are close to graduate from their professional training. Meanwhile, the analysis carried out is correlated to the specific competences of the training of graduates; competences that are framed in: Teaching, Evaluating and Training; components of a professional in education and with specificity in basic education. A comparison is made between the program, the institution, the headquarters and the reference group, which made it possible to consolidate a descriptive study and an approximation to the results obtained by the students of the program.

\section{KEYWORDS}

Standardized Tests, Generic Competences,
Specific Competences, Basic Education, Graduate

\section{INTRODUCCIÓN}

El propósito de la investigación es la de realizar un estudio de percepción de los resultados obtenidos de las pruebas Saber Pro, en el programa de Licenciatura en Educación Básica con énfasis en Matemáticas, Humanidades y Lengua Castellana, para el año 2020, de la Universidad Pedagógica y Tecnológica de Colombia. Se logró la apreciación del análisis de los resultados, mediante la representación gráfica y estadística de los mismos; además del soporte conceptual de distintos autores y teorías que perfilan un debate epistemológico de las pruebas estandarizadas en Colombia, específicamente de las pruebas Saber Pro, como objeto de evaluación de los estudiantes de los programas profesionales de pregrado en las instituciones de Educación Superior. Lo anterior, permitió realizar una fundamentación teórica de los exámenes estandarizados, que pretenden medir la calidad educativa, mediante una connotación homogénea para una población heterogénea, en condiciones y oportunidades de formación muy distantes a una educación con igualdad y equidad.

\section{¿QUÉ SON LAS PRUEBAS SABER PRO?}

Actualmente en Colombia, se aplican pruebas estandarizadas internacionalmente, como la prueba PISA para medir la calidad de la educación básica del país. Resultados, que no han sido los mejores, según informes de los últimos años de la Organización para la Cooperación y el Desarrollo Económico (OECD, 2018), en los cuales se publica que Colombia ocupa los últimos lugares, aunque también se destaca las políticas del gobierno que se han generado en la última decana con el propósito de amortiguar las dificultades económicas y sociales de la población con menos oportunidades y así impactar en el mejoramiento de los resultados 
de evaluación en la calidad educativa de la educación básica del país.

Por otra parte, Colombia aún no evalúa los procesos de enseñanza y aprendizaje en la educación superior por medio de un examen estandarizado Internacionalmente, pero si lo hace localmente por medio de las pruebas Saber Pro (Carrascal y Giraldo, 2019). Entonces, al hablar de las Pruebas Saber Pro, se hace necesario mencionar que sus resultados contribuyen al mejoramiento de los procesos de formación superior en las universidades. En tanto el sistema de evaluación de las instituciones de educación superior se constituye a partir de dos ejes: la autoevaluación institucional y las pruebas estandarizadas estatales llamadas inicialmente Examen de Calidad de la Educación Superior (ECAES), hoy Pruebas Saber Pro (Bogoya, 2013).

Un aspecto interesante en lo refiere a la dinámica de los exámenes académicos de estado para la medición de la calidad de los procesos de formación en las universidades en Colombia, tiene que ver con la aplicación e importancia de las Pruebas Saber Pro, para obtener un título profesional en el país. Es así como, el Ministerio de Educación Nacional- MEN, concibe en definir estos exámenes como pruebas académicas de carácter oficial y obligatorio, denominadas genéricamente, Pruebas Saber Pro, las cuales, forman parte de un conjunto de instrumentos que el estado colombiano dispone para evaluar la calidad de la educación formal recibida por quienes culminan los distintos programas académicos profesionales en las Instituciones de Educación Superior-IES (Ministerio de Educación Nacional - MEN, 2009).

\section{IMPORTANCIA DE LAS PRUEBAS SABER PRO EN COLOMBIA}

El análisis de los resultados que se visibilicen de las pruebas Saber Pro, tienen un alto impacto en las universidades, generando cambios en los currículos y su forma de evaluar. También en la forma como la universidad prepara a los estudiantes para presentar la prueba, fija parámetros y criterios para organizar el sistema de evaluación. (Vega, 2017). Por cuanto, es un estrategia implementada por el gobierno, para identificar los niveles de calidad educativa, además de establecer planes de mejoramiento que contribuyan a superar las dificultades y los bajos porcentajes que se evidencien en el desarrollo de competencias profesionales, las cuales impactan directamente con el avance social, industrial y económico del país.

Además, se ha identificado que las Pruebas Saber Pro, constituyen un referente en la medición para la universidad en un ámbito local y nacional, de tal manera que es una forma de posicionar a las instituciones de educación superior, en el ranking nacional que determina la calidad de las mejores universidades del país. Al respecto, Mesa (2009), afirma que, para la pruebas Saber Pro los factores que inciden en los resultados contribuyen a mejorar la calidad académica y generar procesos de autoevaluación a fin de mejorar también, en competencias como razonamiento cuantitativo, lectura crítica, inglés y comunicación escrita. En este sentido, según lo contemplado en el Decreto 3963 de octubre de 2009, del Ministerio de Educación Nacional de Colombia- MEN, uno de los objetivos del Saber Pro es comprobar el grado de desarrollo de las competencias de los estudiantes próximos a culminar los programas académicos de pregrado que ofrecen las instituciones de educación superior.

Tobón (2008), define las competencias como procesos complejos de desempeño con idoneidad en determinados contextos, integrando diferentes saberes (saber ser, saber hacer, saber conocer y saber convivir), para realizar actividades $y / 0$ resolver problemas con sentido de reto, motivación, flexibilidad, creatividad, comprensión y emprendimiento. Así 
pues, teniendo en cuenta la importancia de la adquisición de competencias para el desempeño del rol profesional dentro de la sociedad, también se hace relevante conocer los factores que inciden en determinar este nivel de adquisición. La evaluación medida a través de la Pruebas Saber y la trascendencia de sus resultados, favorece la generación de cambios estructurales del currículo, la enseñanza y los modelos educativos, en pro del avance en la calidad de los programas y por ende un mejor desempeño integral de sus egresados, demostrando la formación académica, profesional y axiológica recibida en la universidad.

Lo anterior, se contrasta con la poca atención que se da a la evaluación formativa al interior de las aulas, y la relevancia que tienen los exámenes estandarizados. Los resultados de las Pruebas Saber Pro, son un elemento importante para medir la calidad educativa de las universidades; la Ley 1324 de 2009, confiere al Instituto Colombiano para Evaluación de la Educación-ICFES, la misión de evaluar, mediante exámenes externos estandarizados, la formación que se ofrece en el servicio educativo en los distintos niveles. También establece que el MEN, define lo que debe evaluarse en estos exámenes (ICFES, 2014). En la actualidad, el ICFES diseña y aplica las pruebas Saber Pro, con las cuales se evalúa la Educación Superior. En palabras de la normatividad colombiana, la evaluación de la educación superior, está medida por los resultados obtenidos en las Pruebas Saber Pro, y su impacto debe generar cambios profundos al interior de la estructura académica y pedagógica de los programas, de tal manera que conlleven a la actualización de procesos que respondan con asertividad a las demandas de los profesionales en el mercado laboral.

Igualmente, el Decreto 1290 de 2009 propuesto por el MEN, establece que un propósito de la evaluación institucional es el de identificar las características personales, intereses, ritmos de desarrollo y estilos de aprendizaje del estudiante para valorar sus avances. Por consiguiente, sería coherente pensar que, con estos mismo propósitos han sido diseñas las Saber Pro, entendiendo que las competencias generales valoradas son las Competencias Ciudadanas, Comunicación Escrita, Inglés, Lectura Crítica y Razonamiento Cuantitativo. Sin embargo, es difícil imaginar que con la estandarización de estas competencias bajo el modelo de las pruebas aplicadas, se logre establecer una conexión directa con la normatividad del Ministerio, en la cual se visiona una individualización del aprendizaje de cada estudiante.

Las pruebas Saber Pro, de obligatoria presentación por parte de los futuros graduandos universitarios en Colombia, se erigen como un posible modelo de medición de la calidad docente de los diferentes programas de pregrado del país. (Boada y Cardona Montoya, 2017). Entonces, surge como producto de esta afirmación, que son tan importantes los resultados de las Saber Pro, como lo son las estrategias metodológicas y pedagógicas que se desarrollan en cada programa, lo que en su conjunto constituye en palabras de Boada y Cardona, básicamente, el examen general para medir el rendimiento de los futuros profesionales del país; donde la competencias académicas hacen referencia a la aplicación de los conocimientos adquiridos en el pregrado. Así pues, la evaluación de estas competencias implica exactamente, la descripción de contextos, cuya apropiación está directamente relacionada con la naturaleza del pregrado al cual pertenece el estudiante.

De lo anterior se desprende que, existe un marcado interés de IES en Colombia, por mejorar el desempeño académico de los estudiantes que presentan estos exámenes, debido a que los resultados obtenidos en las mismos, son tenidos en cuenta como indicadores que 
inciden directamente en la medición que realiza el MEN, a través del ICFES, y cuyo impacto se evidencia significativamente en todos los procesos académicos y de formación de dichas Instituciones (García-González, SánchezSánchez, Orozco y Obredor, 2019). De acierta forma, las universidades se ven comprometidas en elevar los resultados obtenidos en las Saber Pro, porque son la evidencia tangible que se tiene ante el Ministerio de Educación, de la labor cumplida según las exigencias y normatividad vigente para el desarrollo de procesos formativos en cada uno de los programas ofertados y que están vigilados y avalados por esta misma entidad, en pocas palabras los resultados muestran el deber cumplido de la universidad con sus educandos.

\section{CALIDAD DE LA EDUCACIÓN Y LA EVALUACIÓN}

La evaluación de la calidad educativa en Colombia, es un tema que se ha discutido en múltiples escenarios. No es sencillo establecer las estrategias que permitan determinar el nivel de asociación que existen entre los resultados de la evaluación educativa y la calidad de las intervenciones pedagógicas y didácticas que se desarrollan al interior de las aulas de clase. Sin embargo, hacer estudios que señalen los resultados obtenidos de las pruebas estandarizadas, es importante para las instituciones educativas, por cuanto esto conlleva a la identificar elementos claves para mejorar en los procesos académicos de tal manera que se reflejen en mejores resultados de evaluación.

Yepes (2010), explica que la evaluación puede servir como un motivador para el desempeño de los estudiantes. Si estos aprenden a valorar la evaluación como indicador de desempeño, se esforzaran por lograr cada vez un mejor rendimiento. Posiblemente, los procesos de evaluación no siempre serán bien recibidos para quien es evaluado, sin embargo, cuando exista a la par, elementos de realimentación, en los cuales se involucren docentes, estudiantes y demás actores decisivos en el proceso educativo, es factible que pueda lograrse un cambio de actitud y de resiliencia que fomente la motivación y el interés por aprender y superar las fallas cometidas; de tal forma que se convierta en una oportunidad para crecer en lo personal y lo académico, en busca de la idoneidad, como característica principal de la adquisición de competencias de desempeño integral del individuo.

Fernández (2005), afirma que es función principal de la evaluación en la educación, orientar y apoyar las acciones de mejoramiento de la calidad mediante la obtención, análisis e interpretación de información válida y confiable. En tanto, las formas de evaluación, que incluyan desde su diseño una incorporación de los avances en el campo de las ciencias de educación, la cognición, la pedagogía, aportaría elementos claves para la toma de decisiones, de manera acertada en los ámbitos educativos, en los cuales intervienen de manera inherente la enseñanza, el aprendizaje, la formulación de proyectos, la definición de modelos y el perfeccionamiento de los procesos curriculares y didácticos, propios de la gestión y desarrollo de la formación profesional en las universidades e instituciones de educación superior.

Sin embargo, hablar de calidad enmarcada dentro de los procesos estandarizados de evaluación, no es del todo la realidad infinita y el reflejo de lo que pasa con la formación en las universidades; la evaluación no solo es cuestión de las pruebas estandarizadas y la calidad no solo es el resultado de las mismas, es apenas uno de los aspectos en el sistema educativo y del macro proceso educativo, no solo se puede colocar la atención a los resultados de las pruebas estandarizadas nacionales e internacionales, sino que se ha de analizar el sistema en general para avanzar hacia la calidad de la educación 
con la ayuda de la evaluación (CifuentesMedina, Chacón-Benavides y Fonseca-Correa; 2018). Sin embargo, Ayala Sánchez (2017), afirma al respecto que, una forma de medir la calidad de la educación superior es a través de los resultados de la evaluación en competencias de los estudiantes, que para el caso de Colombia se hace mediante el instrumento conocido como examen Saber Pro.

A su vez, los resultados de las Saber Pro transcienden, ya que generan indicadores por medio de los cuales es posible comparar conceptos y categorías que han modificado la manera de percibir los procesos educativos. La sociedad actual plantea a la educación múltiples retos que impulsan a la comunidad académica a investigar y profundizar los conocimientos frente a los fenómenos y determinantes asociados a la calidad de la educación (Castro Ávila, y Ruiz Linares, 2019).

\section{MATERIALES Y MÉTODOS}

La investigación se enmarca en el enfoque mixto no experimental de tipo descriptivo, puesto que da cuenta de los resultados de las pruebas Saber Pro de los estudiantes del programa de Licenciatura en Educación Básica con Énfasis en Matemáticas, Humanidades y Lengua Castellana para año 2020. Es población heterogénea, compuesta por personas entre 18 y 27 años, siendo el porcentaje de mujeres mayor que el de hombres.

La Tabla No. 1, describe la muestra seleccionada para es estudio, la cual contó con la participación de estudiantes matriculados de noveno y décimo semestre del programa. El objeto de la investigación, proviene de diferentes regiones del país en donde se tiene la oferta académica.
Tabla $\mathbf{N}^{\circ}$. 1. Muestra en función del género de los estudiantes participantes

\begin{tabular}{|l|c|c|}
\hline Género & Muestra & Porcentaje \\
\hline Femenino & 240 & $75 \%$ \\
\hline Masculino & 80 & $25 \%$ \\
\hline TOTAL & 320 & $100 \%$ \\
\hline
\end{tabular}

Fuente: Elaboración propia

La población del estudio comprende 320 estudiantes de los Centros Regionales de Educación a Distancia - CREAD de: Tunja, Duitama, Sogamoso, Chiquinquirá y Bogotá; que presentaron las pruebas Saber Pro. Una vez caracterizado el grupo, se evidencia que la mayoría de estudiantes son de género femenino con un $75 \%$ y el restante $25 \%$ de género masculino.

Se obtienen los resultados de la prueba, en conjunto y de manera global de los estudiantes del programa de Licenciatura, de las bases de datos del sistema del Instituto Colombiano para el Fomento de la Educación Superior -ICFES. La información del ICFES, es usada para realizar un análisis descriptivo de los desempeños de los estudiantes del programa de Licenciatura en Educación Básica con Énfasis en Matemáticas, Humanidades y Lengua Castellana, el cual se ofrece en modalidad a distancia con apoyo virtual en la Facultad de Estudios a Distancia de la Universidad Pedagógica y Tecnológica de Colombia; se canaliza la oferta a través de los Centros Regionales de Educación a Distancia -CREAD, en diferentes departamentos y ciudades de Colombia.

Gracias al consentimiento informado, de la población de estudio, se realizó el proceso de sistematización de la información, consignada a la Universidad de manera global; de los estudiantes que presentaron la prueba Saber Pro, en el año 2020, y perteneciente a la Licenciatura en Educación Básica con énfasis en Matemáticas, Humanidades y Lengua Castellana. 


\section{DISCUSIÓN DE RESULTADOS}

El análisis estadístico descriptivo de las pruebas que presentaron los estudiantes del Programa, en el 2020, está enmarcado desde dos aristas que a su vez apuntan a una misma dirección. En primer lugar se presentan y analizan los resultados de las Competencias Genéricas y, por otro lado, se presenta los resultados y análisis de los módulos específicos en educación para el mismo año 2020.

\section{ANÁLISIS DESCRIPTIVO DE LAS COMPETENCIAS GENÉRICAS DEL FUTURO DOCENTE}

Los resultados de las competencias genéricas que corresponden a Comunicación Escrita, Razonamiento Cuantitativo, Lectura Crítica, Competencias Ciudadanas e Inglés; conllevan a evidenciar las habilidades, conocimientos y actitudes que los futuros profesionales han adquirido para desempeñarse en su rol propio de la formación adquirida en la universidad, correlacionado directamente con la sede de la institución y el grupo de referencia.

Gráfica $\mathbf{N}^{\circ}$ 1. Promedio del puntaje global 2020

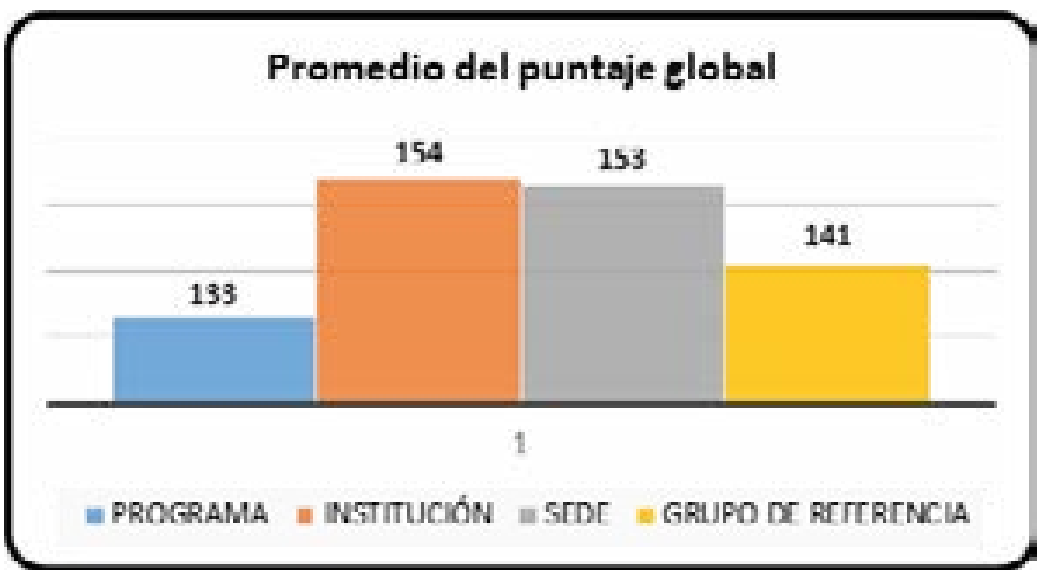

Fuente: Elaboración propia

Según los niveles de agregación se evidencia que, los desempeños del programa de Licenciatura en Educación Básica, están por debajo del promedio institucional que alcanza un promedio superior a la media. En este mismo sentido, se puede apreciar que el promedio del programa está también por debajo del promedio del grupo de referencia del núcleo básico común de conocimiento a nivel de todas las licenciaturas que ofertan programas de licenciatura de las diferentes universidades del país.

En Colombia se ha dado una evolución histórica notable de las pruebas estandarizas aplicadas en los diferentes niveles educativos. Para Educación Superior, la creación de las pruebas
ECAES en el 2002, comienza a establecer su proceso de evaluación; luego éstas denominadas posteriormente pruebas Saber Pro. Cabe destacar que, la transformación del proceso es evidente y significativo, tanto de en su estructura como en el trasfondo, ya que se pasa de evaluar conocimientos a evaluar competencias, y adicionalmente la evaluación de competencias se establecer en dos categorías esenciales: Competencias genéricas (dirigidas a evaluar el desempeño laboral de todo profesional) y Competencias específicas (en las que se establece la evaluación de las actitudes, habilidades y desempeños propios de 
una profesión en su quehacer especifico de la disciplina de estudio).

Para los profesionales de la educación, las pruebas Saber Pro, evalúan las competencias genéricas y específicas, tal como lo establece el ICFES. Así, para el año 2020, el examen trae una sesión obligatoria de preguntas, para todos los que presentan la prueba, la cual se compone de cinco (5) módulos que evalúan las ya mencionadas competencias genéricas
(Lectura crítica, Razonamiento cuantitativo, Competencias ciudadanas, Comunicación escrita e Inglés).

La información de la Gráfica $\mathrm{N}^{\circ} 2$, evidencia los resultados comparativos entre programa, institución, sede y grupo de referencia. Para el módulo de comunicación escrita los resultados del programa están por debajo del promedio de la institución, la sede y así mismo está por debajo del grupo de referencia y en el módulo de razonamiento cuantitativo los desempeños de los estudiantes del programa.

Gráfica $N^{\circ} 2$. Promedio por módulos de competencias genéricas

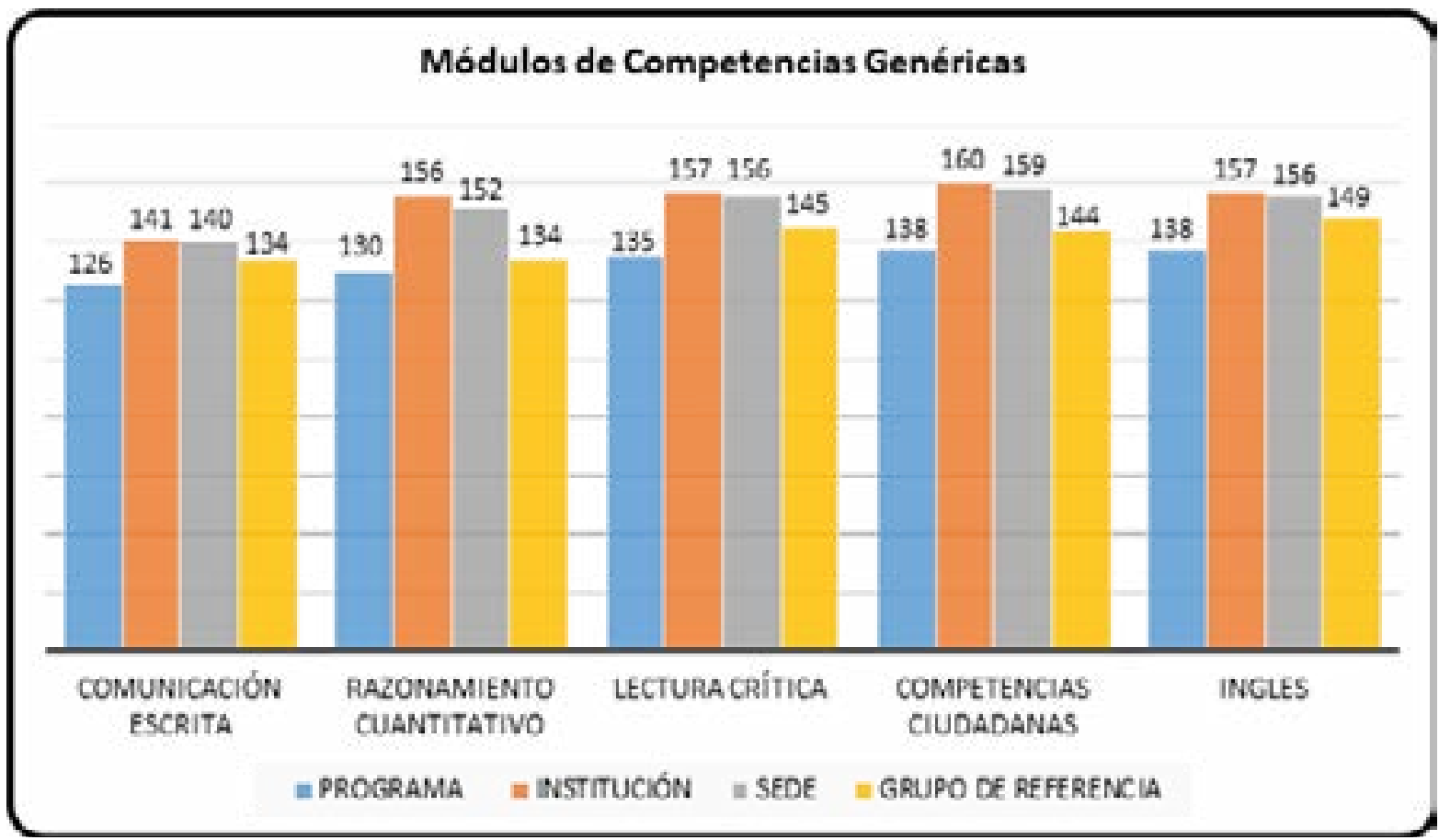

Fuente: Elaboración propia

Con relación al módulo de lectura crítica, los desempeños de los estudiantes del programa se encuentran también por debajo de los desempeños de la institución y del grupo de referencia y en el módulo de competencias ciudadanas se aprecia que, el desempeño de los estudiantes del programa está por debajo del promedio de la institución, de la sede y del grupo de referencia. Sin embargo, en este módulo se ha presentado un leve ascenso con relación a los anteriores años. Por último, los desempeños en el módulo de inglés están por debajo de los promedios de los grupos de referencia, así como también, por debajo de los promedios de la sede y de la institución. Así mismo, se tiene que con relación a los resultados de los últimos años, 
los desempeños en este módulo han mejorado levemente.

Según Timarán-Pereira, S. R., et al. (2016);

Uno de los objetivos del examen de Estado de calidad de la educación superior Saber Pro, según el Ministerio de Educación Nacional MEN, 2009, a través del Decreto 3963 del 14 de octubre del 2009, es el de comprobar el grado de desarrollo de competencias de los estudiantes próximos a culminar los programas académicos de pregrado que ofrecen las instituciones de educación superior. El examen está compuesto por pruebas que evalúan competencias genéricas y específicas (p. 15)

En consecuencia, los aspectos de la normatividad subsiguiente a 2009, pasaron de evaluar conocimientos a competencias al igual que la organización de la estructura de la prueba. Para el 2020, el examen está organizado para medir la lectura crítica, el razonamiento cuantitativo, las competencias ciudadanas, la comunicación escrita y el inglés; dirigido a todos los estudiantes de los diferentes programas de pregrado profesional, que están próximos a terminar el pensum académico del programa que cursan.

Se disgrega en el análisis y apreciación de los resultados de las pruebas Saber Pro, del programa académico objeto del presente estudio, que una posible afectación del bajo desempeño puede estar asociada a las consecuencias de la pandemia generada por coronavirus SARS-CoV-2 (COVID-19) y las implicaciones que esto condujo al cambio para presentar las pruebas, pues la modalidad pasó del ser presencial a semi presencial y virtual. Además, a todos los cambios y coyunturas por las cuales atravesó la educación en Colombia, que debieron ser atendidas en el transcurrir del año 2020; los cuales en Colombia al igual que en muchas partes del mundo, han derivado transformaciones profundas en los paradigmas de todos los contextos y escenarios de la vida cotidiana, que desde luego involucran también a los estudiantes de la Licenciatura en Educación Básica con énfasis en Matemáticas Humanidades y Lengua Castellana, que son objeto de la muestra tomado para la realización del presente análisis.

Gráfica º3. Promedio módulos de competencias genéricas

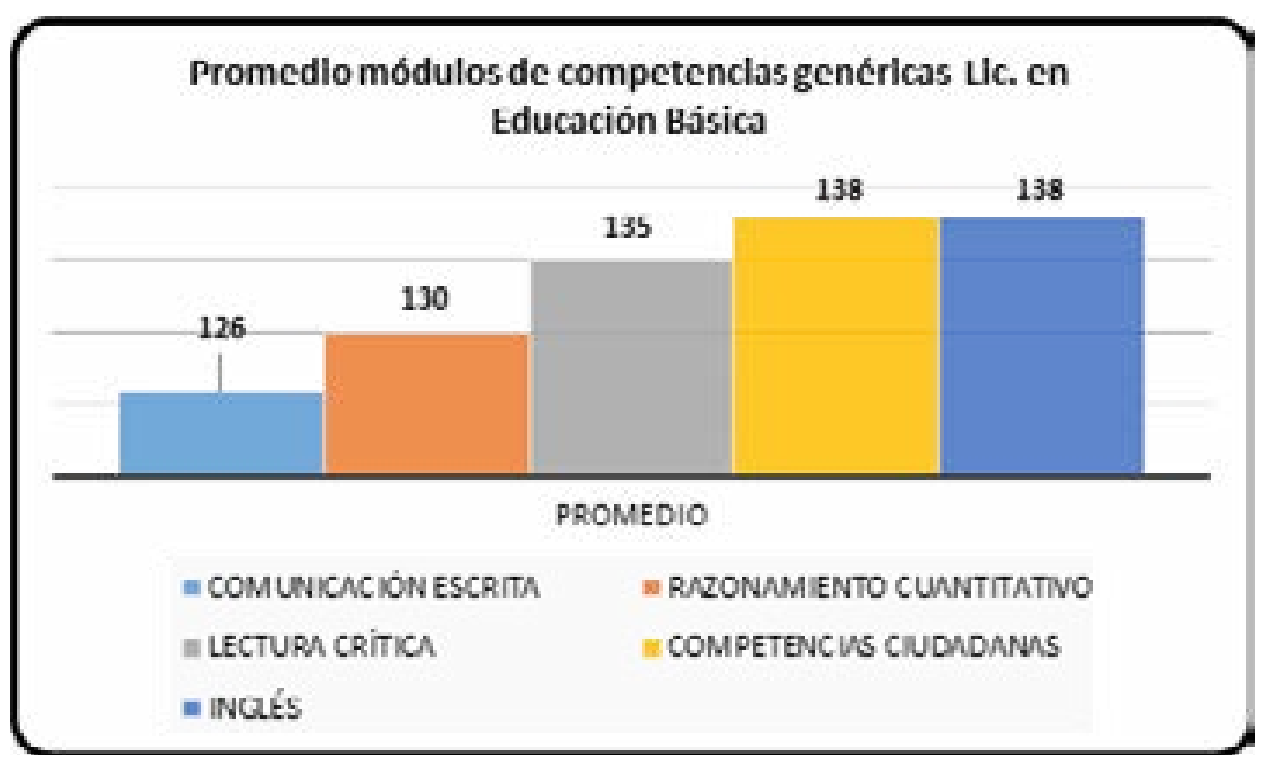

Fuente: Elaboración propia 
De acuerdo con la información que se muestra en la Gráfica $N^{\circ} 3$, los mejores desempeños de los estudiantes en cada uno de los módulos de competencias genéricas, se tienen en las competencias ciudadanas e inglés con una puntuación de 138 en promedio; seguidos del módulo de lectura crítica y razonamiento cuantitativo, con un puntaje de 135 y 130 respectivamente. Finalmente, está el módulo de comunicación escrita obteniendo el menor puntaje con un valor de 126.

Para el año 2020, en el programa de Licenciatura en Educación Básica con énfasis en Matemáticas, Humanidades y Lengua Castellana, de la UPTC, población objeto de estudio, se evidencia el posicionamiento de los módulos de Competencias Ciudadanas e inglés con relación a los demás módulos. Sin embargo se destaca que no hay una diferencia notable, frente a la relación del programa con la institución, sede y grupo de referencia.

280 Los módulos de Competencias genéricas (Comunicación Escrita, Razonamiento Cuantitativo, Lectura Crítica, Competencias Ciudadanas e inglés); tienen por objetivos: comprobar el grado de desarrollo de las competencias de los estudiantes, producir indicadores de valor agregado y servir de fuente de información para la construcción de indicadores de evaluación de la calidad de los programas e instituciones de educación superior por lo tanto Timarán-Pereira, S. R., et al. (2016) afirma que;

A pesar que en la prueba Saber Pro no se pretende que los estudiantes de todas las carreras profesionales desarrollen las competencias genéricas a un mismo nivel, ni aún las comunes a grupos de programas, sí es importante para el sector de la educación superior conocer cómo se asocian los factores correspondientes a las dimensiones sociodemográficas, económicas, académicas e institucionales del estudiante en relación con el nivel de desempeño en estas competencias según las pruebas Saber Pro (p. 15).

Los programas e instituciones inmersos en los indicadores y en el proceso de evaluación por cuenta de las pruebas Saber Pro, han comenzado a establecer planes de mejora, diversas formas de preparación y estímulos a los estudiantes de mejores resultados para captar su atención y así obtener una adecuada preparación, más allá de ser considerado como solo requisito de grado.

\section{ANÁLISIS DESCRIPTIVO DE LAS COMPETENCIAS ESPECÍFICAS DEL FUTURO DOCENTE}

El Instituto Colombiano para la Evaluación de la Educación -ICFES, presenta los objetivos de evaluación, la estructura y el contenido de los módulos específicos: Enseñar, Formar y Evaluar, que hacen parte del Examen de Estado para la Evaluación de la Educación Superior - Saber Pro. Además señala que, el objetivo principal consiste en responder las siguientes preguntas: ¿qué competencias se evalúan en los módulos específicos de Enseñar, Formar y Evaluar? y ¿cómo se evalúan? (ICFES, 2018).

Estas tres competencias específicas, Enseñar, Formar y Evaluar, son transversales y se vinculan directamente con el currículo que integra la formación profesional de los docentes 
Tabla $\mathrm{N}^{\circ}$ 2. Competencias específicas de los profesionales en Educación

\begin{tabular}{|c|l|}
\hline COMPTENCIA & \multicolumn{1}{|c|}{ BREVE DESCRIPCIÓN } \\
\hline Enseñar & $\begin{array}{l}\text { Competencia para comprender, formular y usar la didáctica de las } \\
\text { disciplinas con el propósito de favorecer los aprendizajes de los } \\
\text { estudiantes }\end{array}$ \\
\hline Formar & $\begin{array}{l}\text { Competencia para reconceptualizar y utilizar conocimientos } \\
\text { pedagógicos que permitan crear ambientes educativos para el } \\
\text { desarrollo de los estudiantes, del profesor y de la comunidad. }\end{array}$ \\
\hline Evaluar & $\begin{array}{l}\text { Competencia para reflexionar, hacer seguimiento y tomar decisiones } \\
\text { sobre los procesos de formación, con el propósito de favorecer la } \\
\text { autorregulación y plantear acciones de mejora en la enseñanza, en el } \\
\text { aprendizaje y en el currículo. }\end{array}$ \\
\hline
\end{tabular}

Fuente: Elaboración propia- Información Recabada ICFES 2018

En las especificaciones que establece el ICFES refiere a seis competencias comunes e interconectadas que orientaban la formulación de las propuestas curriculares de los programas de formación inicial en las facultades de educación. Programas entre los cuales se encuentra la Licenciatura en Educación Básica 281 con énfasis en Matemáticas, Humanidades y Lengua Castellana.

Las seis competencias comunes a las cuales se hace mención son: Saber qué es, cómo se procesa y para qué son los conocimientos objeto de la práctica educativa; saber enseñar los conocimientos objeto de la práctica educativa; saber organizar y desarrollar ambientes de aprendizaje, saber evaluar las enseñanzas, los aprendizajes y el currículo; saber proponer, desarrollar y evaluar proyectos educativos y de aula y, saber contextualizar la práctica pedagógica.

Así pues, se establece una relación estrecha entre las seis competencias comunes y las tres competencias específicas afines a los programas de los cuales hacen parte estudiantes de la educación, entre los cuales se encuentra el programa objeto de estudio; que fueron evaluados para el año 2020, obteniendo los resultados que se evidencian en la gráfica $\mathrm{N}^{\circ} 4$.

Gráfica $N^{\circ} 4$. Promedios competencias especificas

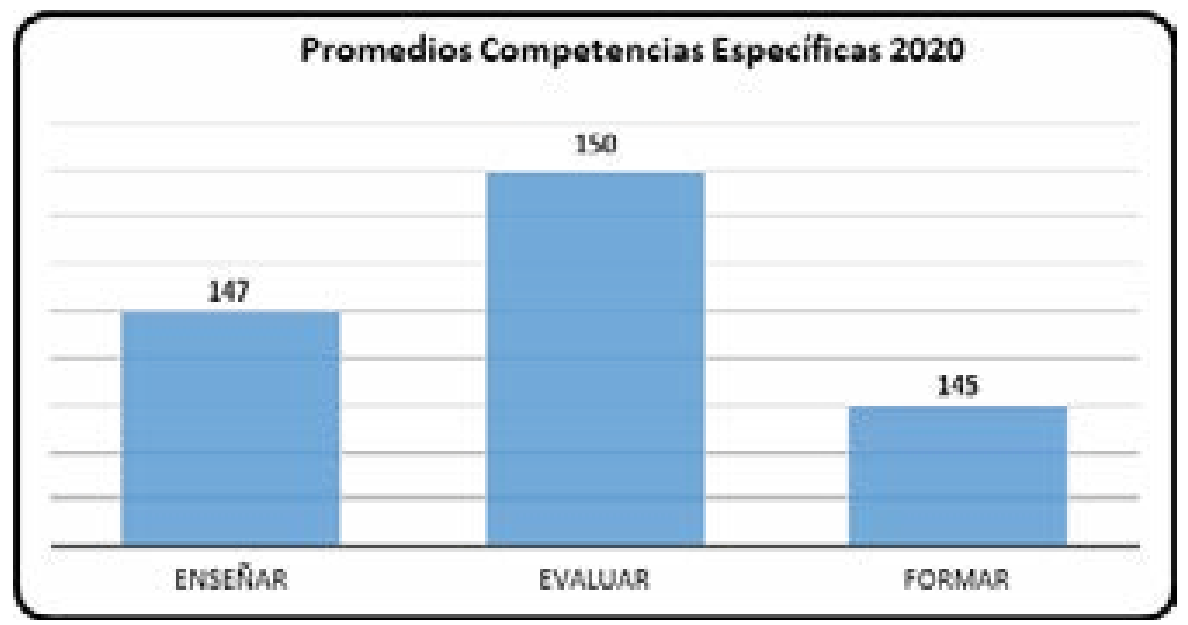

Fuente: Elaboración propia 
La Gráfica $\mathrm{N}^{\circ} 4$, presenta los desempeños de los estudiantes en los módulos de competencias epecificas propios del futuro docente, se puede apreciar que el módulo de mejor desempeño es el de Evaluar con un valor de 150, seguido del módulo Enseñar de 147 y por último el módulo Formar con 145 puntos.
Los resultados de las competencias específicas de los estudiantes del programa de Licenciatura en Educación Básica con énfasis en Matemáticas Humanidades y Lengua Castellana para el año 2020 están por encima de los resultados obtenidos en las competencias genéricas, logrando así un mejor desempeño en su área propia de formación como futuros profesionales de la educación.

Gráfica № 5 . Promedios Comparativos competencias especificas

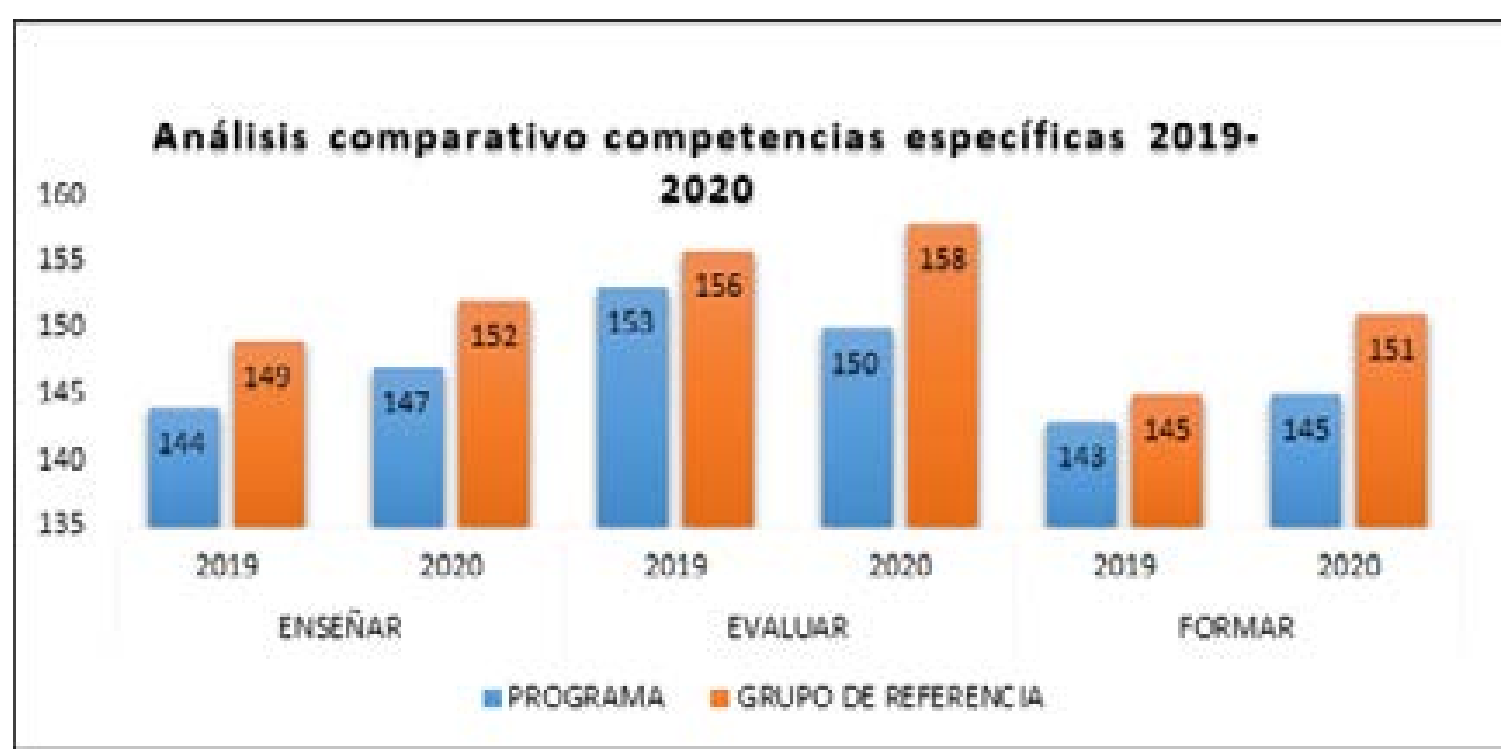

Fuente: Elaboración propia

De acuerdo con la información presentada en la gráfica 5 donde se realiza un comparativo de los años 2019 y 2020 en la prueba de competencias específicas, se puede observar que los estudiantes del programa tuvieron un mejor desempeño en el módulo Evaluar en el año 2019, aun estando por debajo del promedio del grupo de referencia. Así mismo los desempeños de los estudiantes del programa en los módulos Enseñar y Formar están por debajo del promedio del grupo de referencia tanto en el 2019 como en el 2020.

\section{A MANERA DE CONCLUSIÓN}

La calidad de la educación superior en Colombia, se encuentra vigente en los últimos planes y programas del gobierno nacional, aunque algo enredado y poco alcanzable, ya que no ostenta comparación entre los índices de calidad que se pretenden alcanzar con equidad para acceder a ésta, en todos los rincones del territorio geográfico. Se evidencia falta de efectividad para afrontar los retos que se imponen en la evolución en los fenómenos sociales, económicos, culturales y su influencia en el desarrollo educativo y por ende en los resultados de las pruebas nacionalmente estandarizadas. 
Los resultados de los cinco (5) módulos que evaluaron las competencias genéricas para el año 2020; en el programa de Licenciatura en Educación Básica con énfasis en Matemáticas Humanidades y Lengua Castellana de la UPTC, se encuentran por debajo del promedio de la institución, la sede y así mismo están por debajo del grupo de referencia; generando una reflexión consecuente a la situación particular de posible incidencia de la pandemia de Covid19 por las particularidades atípicas con las que se dieron para éste periodo académico.

De las tres competencias analizadas, los estudiantes del programa objeto de estudio obtienen mayor dominio de la competencia de seguimiento a los procesos, toma de decisiones para el mejoramiento, procesos de autorregulación; que corresponde a evaluar. Seguido de los conocimientos pedagógicos, didácticos y disciplinares, planear la enseñanza, desarrollar la clase, reflexionar sobre los propósitos de la práctica docente; en concordancia con enseñar. Por último, se destacan en promover el desarrollo de sus estudiantes, promover el desarrollo profesional del maestro, promover el desarrollo de la comunidad educativa; asociada a la competencia de formar.

Entre las competencias anteriormente mencionadas, la diferencia solo refiere a cinco (5) porcentuales, razón por la cual se considera necesario fortalecer en el currículo del programa con una visión holística y fundada en la esencia de la configuración del futuro ejercicio profesional como docentes de educación básica.

\section{REFERENCIAS BIBLIOGRÁFICAS}

Ayala Sánchez, Y. (2017). Análisis descriptivo de los resultados de las pruebas Saber Pro en la UPTC del periodo 2012-2015. Pensamiento $Y$ Acción, (22), 59-77. Recuperado a partir de https://revistas.uptc.edu.co/index.php/ pensamiento_accion/article/view/7399

Boada, A., y Cardona Montoya, G. (2017). Análisis comparativo del desempeño de estudiantes de las modalidades, presencial y virtual en las pruebas saber pro Competencias Generales-. Caso de la IES CEIPA.

Bogoya, D. (2013). Educación Superior en Colombia. Universidad Nacional.

Carrascal, A. I. O. y Giraldo, J. J. (2019). Minería de datos educativos: Análisis del desempeño de estudiantes de ingeniería en las pruebas SABERPRO. Revista Politécnica, 15 (29), 128-140. DOI: https://doi.org/10.33571/ rpolitec.v15n29a10

Castro Ávila, M., y Ruiz Linares, J. (2019). La educación secundaria y superior en Colombia vista desde las pruebas Saber. Praxis \& Saber, 10(24), 341-366.

Cifuentes-Medina, J. E, Chacón-Benavides, J. A. y Fonseca-Correa, L. Á. (2018). Análisis del desempeño de los estudiantes de licenciatura en educación básica en las pruebas Saber Pro. Revista de Orientación Educacional, 32(62), 21-46.

Colombia, Instituto Colombiano para la Evaluación de la Educación, ICFES. (2014). Alineación del examen SABER $11^{\circ}$. Lineamientos generales $2014-$ 2 Sistema Nacional de Evaluación Estandarizada de la Educación. Bogotá, Colombia. 
Colombia, Ley 1324 de 2009. Por la cual se fijan parámetros y criterios para organizar el sistema de evaluación de resultados de la calidad de la educación, se dictan normas para el fomento de una cultura de la evaluación, en procura de facilitar la inspección y vigilancia del Estado y se transforma el ICFES.

Colombia, Ministerio de Educación Nacional, MEN. Decreto 1290 de 2009. Por el cual se reglamenta la evaluación del aprendizaje y promoción de los estudiantes de los niveles de educación básica y media.

Colombia, Ministerio de Educación Nacional, MEN. Decreto 3963 de 2009. Por el cual se reglamenta el Examen de Estado de Calidad de la Educación Superior.

Fernández, H. (2005). Cómo interpretar la evaluación pruebas Saber. Subdirección de Estándares y Evaluación. Bogotá, Colombia. Ministerio de Educación Nacional.

García-González, José R., SánchezSánchez, Paola A., Orozco, Manuel, y Obredor, Sergio. (2019). Extracción de Conocimiento para la Predicción y Análisis de los Resultados de la Prueba de Calidad de la Educación Superior en Colombia. Formación universitaria, 12(4), 55-62. https:// dx.doi .org/10.4067/S0718 50062019000400055

Mesa, H. (2009), De los ECAES, la autonomía universitaria y el derecho, Revista Electrónica.

OECD. (2018). Resultados del Programa para la Evaluación Internacional de Alumnos (PISA) en Colombia.
Tobón, S. (2008). La formación basada en competencias en la educación superior: el enfoque complejo. México: Universidad Autónoma de Guadalajara.

Timarán-Pereira, S. R., Hernández-Arteaga, I., Caicedo-Zambrano, S. J., HidalgoTroya, A. y Alvarado Pérez, J. C. (2016). Descubrimiento de patrones de desempeño académico con árboles de decisión en las competencias genéricas de la formación profesional. Bogotá: Ediciones Universidad Cooperativa de Colombia. Doi: http://dx.doi. org/10.16925/9789587600490

Vega, J. A. B. (2017). Factores Demográficos, Académicos y Socioeconómicos que Influyen en los Resultados del Componente Genérico de la Prueba Saber Pro (Doctoral dissertation, Universidad Pedagógica y Tecnológica de Colombia.

Yepes, R. L. G. (2010). Calidad Educativa: Más que resultados en pruebas estandarizadas. Revista Educación y Pedagogía, 16(38). 